\title{
- \\ Overdiagnosis paradigm: not suitable for decreasing the overuse of antibiotics
} OPEN ACCESS

\section{Gloria Cordoba, ${ }^{\oplus}$ Carl Llor}

10.1136/bmjebm-2018-111146

Center for Research and Education in General Practice, Institute of Public health, University of Copenhagen, Copenhagen, Denmark

Correspondence to: Dr Gloria Cordoba, Center for Research and Education in General Practice, Institute of Public health, University of Copenhagen, København, 1014,Denmark; gloriac@ sund.ku.dk

\section{Abstract}

This paper summarises the discussion during the workshop on 'Overdiagnosis and overtreatment of infectious diseases in general practice. How and where to break the endless loop?' The workshop was organised as part of the overdiagnosis conference carried out in August 2018 in Copenhagen, Denmark. During the workshop, participants from all over the world reflected on the challenges of embracing the overdiagnosis paradigm as a tool to advance understanding and to find solutions to the unnecessary use of antibiotics in primary health care. It was concluded that the narrow view of the overdiagnosis paradigm does not provide theoretical resources to address the problem of the inherent connection between diagnostic error and treatment decision. In contrast to the overdiagnosis paradigm, the too much medicine paradigm is an umbrella term suitable to frame actions that recognise the complexity of the decision-making process during the consultation and its impact on overtreatment.

\section{Introduction}

Unnecessary and excessive use of antibiotics is the main driver of antimicrobial resistance. ${ }^{1}$ The prospect of patients dying from common infections caused by multiresistant bacteria in the long term is grim unless the situation is reversed by limiting the use of antibiotics only to patients in whom the benefits outweigh the harms.

Since the publication of the book 'Overdiagnosed: Making People Sick in the Pursuit of Health' by Welch et al in $2011^{2}$ and the launching of the first International conference on overdiagnosis in 2013, overdiagnosis has become a widely used term to address the drivers of 'too much medicine'.

Well-defined concepts are essential to advance understanding and to find concrete solutions to a problem. In that sense, Brodersen et al published a paper about what is and is not overdiagnosis. ${ }^{3}$ In this paper, overdiagnosis is defined as the diagnosis of a condition, that would otherwise not cause symptoms or harm to a patient during his or her lifetime'. They go further by arguing that terms such as overtesting, misdiagnosis and false-positives do not belong to the overdiagnosis paradigm.

This assertion has raised more questions than answers within the clinical and research area regarding infectious diseases and the use of antibiotics. In this paper, we summarise the main points discussed during the workshop 'Overdiagnosis and overtreatment of infectious diseases in general practice. How and where to break the endless loop?'. ${ }^{4}$ During the workshop, participants from all over the world reflected on the challenges of embracing the overdiagnosis paradigm as a tool to advance understanding and to find solutions to the unnecessary use of antibiotics in primary healthcare.

It was concluded that the current definition of overdiagnosis is not suitable and falls short of addressing the complexity of the drivers and consequences of excessive and unnecessary use of antibiotics. On the contrary, the 'too much medicine' paradigm ${ }^{5}$ gives space for a broader understanding of overdiagnosis as errors during the diagnostic process, which are inherently connected to the error in the treatment decision called overtreatment.

\section{Overdiagnosis versus 'too much medicine'}

The current definition of overdiagnosis is based on long-term or chronic conditions and asymptomatic patients. It markedly differs from the type of population managed in primary healthcare due to a suspected acute infectious disease.

First of all, all the patients seeking care at primary healthcare have atypical or typical symptoms, a type of perceived change that triggers the need for seeking healthcare. Second, the focus of benefits against harms is mostly driven by outweighing the benefit of shortening the length of time with symptoms against the harms due to the development of adverse effects including the development of antimicrobial resistance. ${ }^{67}$

Therefore, neither the overdiagnosis of an asymptomatic patient nor the development of unforeseen long-term harms is the main problem regarding the diagnosis and subsequent management of patients with a suspected acute infection.

The main problem is the interplay of multilevel factors that influence the diagnostic process and treatment decision, which leads to overtreatment. ${ }^{8}$ From this perspective, the 'too much medicine' paradigm is an umbrella term that gives room for recognising the singularities of each error while reminding us that they are part of a bigger picture.

Looking for solutions to the unnecessary use of antibiotics is far from being an easy task. Determinants and actions targeting diagnostic errors influence the treatment decision and vice versa. Understanding the components of the bigger picture and the challenges of the diagnostic errors within the 'too much medicine' paradigm are crucial to bringing about effective solutions. 


\section{The bigger picture}

Worldwide, the patient-doctor encounter in primary care faces similar challenges such as (a) short consultation time, (b) high uncertainty of the origin of the symptoms, (c) uncertainty of the evolution of symptoms and (d) organisational challenges for following the patient. ${ }^{9} 10$

During the patient-doctor encounter, patients' expectations and general practitioners' (GP) attitudes toward these expectations are drivers of 'too much medicine'. As mentioned above, there are typical and atypical symptoms that trigger an active search for healthcare. Patients/the family expect something from their GP. It can include a check-up, reassurance, a medical certificate, antibiotic therapy or simply guidance on symptom management.

GPs who perceive high patient expectations for antibiotics are more likely to prescribe antibiotics (ie, overtreatment) unnecessarily. A recent study in Australia found that more than half of the GPs included in the study self-reported that they would prescribe antibiotics for an upper respiratory tract infection to meet patient expectations. ${ }^{11}$ However, while all patients are believed to expect something, only some patients expect antibiotics, and among these patients, a subset demands antibiotics. Notwithstanding, patient expectations may be based on false assumptions, with many overestimating the effectiveness of antibiotic treatment. ${ }^{12}$

It is very important for GPs to meet patients' expectations, as it is a way to preserve a good doctor-patient relationship. Prescribing increases patient satisfaction and reinforces the doctor-patient relationship as clinicians often feel prescribing as less stressful than an anxious patient or parent. ${ }^{13}$ Some clinicians do not feel that it is worth jeopardising their relationship with a patient or parent over a relatively minor matter of prescribing antibiotics. The consequences for the future relationship with patients are more of a concern for GPs prescribing antibiotics than antibiotic resistance. $^{9}$ GPs are generally aware of and concerned about the threat that antimicrobial resistance poses and agree that this is a growing problem. However, antimicrobial resistance is considered more of a public health issue, whereas the GPs' priority is to be responsive to the needs of the individual patient.

This example shows the multidimensional factors that play a role in the decision whether to prescribe antibiotics. There is a patient expecting a solution to his/her problems. There is a GP expecting to be responsive to the needs of the patient. There is a system expecting a rational use of resources and contention of the antimicrobial resistance problem.

\section{The challenges of the diagnostic errors}

Mislabelling or misdefinition, ${ }^{14}$ not considered as overdiagnosis under the definition of overdiagnosis of Brodersen et al, is an error during the diagnostic process. However, it is difficult to know whether mislabelling lead to overtreatment or vice versa. Due to the short time of the consultation in primary care, it is difficult to determine the logical order of the decision taken by the GP. Sometimes a GP mislabels the patient with another diagnosis to better justify the administration of antibiotic therapy, for which treatment is unnecessary. A clear example is the differentiation of a sore throat into two different labels (pharyngitis and tonsillitis). Patients are diagnosed with tonsillitis if the clinicians intend to treat a sore throat with antibiotics and they use the milder category of pharyngitis if they think that the infection has a viral cause. In extreme cases, GPs decide to prescribe antibiotics even before examining the patient. ${ }^{15}$ It goes against logical thinking as treatment should be the subsequent step after a systematic diagnostic process.
It implies that the current system to assess overtreatment is quite inaccurate. Future research should focus on finding better ways to assess overtreatment and the extent of mislabelling. For example, the assessment should be based on the presence of diagnostic information (eg, cluster of signs and symptoms, results of rapid tests) as well as prognostic criteria (eg, age and comorbidities) rather than the label given by the prescriber.

Other examples of the broader vision of errors in the diagnostic process connected to the decision to treat are overtesting and misinterpretation of diagnostic information (ie, false positives). These terms are not considered as overdiagnosis under the Brodersen et al definition of overdiagnosis.

Currently, there is no single item of the diagnostic information (eg, signs, symptoms and results of rapid tests) able to predict whether an infection has a viral or a bacterial origin with $100 \%$ certainty. Systematic reviews about the diagnostic accuracy of signs and symptoms for the most common infections managed in primary care ${ }^{1617}$ have shown that the specificity of signs and symptoms is low. The cluster of symptoms like the Centor criteria-used to predict the bacterial origin of a sore throat ${ }^{18}$-or the cluster of symptoms to identify patients with influenza-like symptoms, ${ }^{19}$ who can benefit from early antiretroviral therapy can increase predictive accuracy, but it depends on the prevalence of the disease. ${ }^{20}$ Thus, a treatment decision only based on signs and symptoms is likely to yield a high proportion of false-positives, resulting in overtreatment.

The use of rapid tests has been advocated as a solution to increase diagnostic accuracy, thereby decreasing overtreatment. ${ }^{21}$ However, the introduction of this technology in primary care is in its infancy with a lot of controversy about the added value. ${ }^{22} 23$

Models to understand the factors influencing the proper use and added value of rapid testing in primary care have demonstrated a complex interaction between availability, interpretation and easy-to-use factors. ${ }^{24}$

Currently, rapid tests to support the diagnostic process in patients with a suspected infection seeking help in primary care are available in very few countries. In most of the world, the decision of whether to prescribe antibiotics is taken based only on signs and symptoms.

One of the few countries with the wide availability of rapid tests is Denmark. This scenario allowed us to assess the use of diagnostic tools in daily practice and its effect on treatment decision in patients with suspected urinary tract infection (UTI). ${ }^{25}$ We found that in $85 \%$ of the patients with a suspected uncomplicated UTI, urine culture was part of the diagnostic process. Many international guidelines recommend the use of urine culture only in patients with suspected complicated UTI; then this $85 \%$ could be regarded as overtesting. However, overtreatment was lower in the group of patients in which urine culture was performed in comparison with the group of patients in which antibiotics were prescribed based only on signs and symptoms. To disentangle the causal path between the use of rapid test and overtreatment is not straightforward. Thus, there is a growing awareness about the fact that the assessment of the causal pathway between the use of a rapid test and the effect on overtreatment needs re-structuring. The field of diagnostic test evaluation requires further development and operationalisation of variables within the "too much medicine' paradigm if the rapid tests are to become systematically introduce in primary healthcare worldwide.

\section{Conclusion}

Overall, it can be seen that the overdiagnosis paradigm is unrelated to these multidimensional factors. The type of population, as 
well as the dilemmas regarding benefits and harms in the management of patients with suspected infections, differ in crucial aspects of the population and dilemmas covered by the overdiagnosis paradigm. The umbrella term of too much medicine is broader and allows the use of theories and strategies without getting trapped into the dilemmas of trying to squeeze the multidimensional factors of diagnostic error and overtreatment into the narrow definition of what is and is not overdiagnosis.

Acknowledgements The authors would like to thank all the participants of the workshop 'Overdiagnosis and overtreatment of infectious diseases in general practice. How and where to break the endless loop?' for sharing their experience about the challenges in everyday practice.

Contributors GC: had the idea for the article. GC and CL: performed the literature search; wrote the article.

Funding This study was in part supported by læge Sofus Carl Emil Friis og Hustru Olga Doris Friis' legat and University Institute in Primary Care Research Jordi Gol.

Competing interests None declared.

Patient consent for publication Not required.

Provenance and peer review Not commissioned; externally peer reviewed.

Open access This is an open access article distributed in accordance with the Creative Commons Attribution Non Commercial (CC BY-NC 4.0) license, which permits others to distribute, remix, adapt, build upon this work non-commercially, and license their derivative works on different terms, provided the original work is properly cited, appropriate credit is given, any changes made indicated, and the use is non-commercial. See: http://creativecommons.org/licenses/by-nc/4.0/.

\section{References}

1. WHO-OIE-FAO. Global framework for development \& Stewardship to combat antimicrobial resistance - draft roadmap. 2017 www.who.int/ medicinedocs/documents/s23198en/s23198en.pdf

2. Welch G, Schwartz LM, Woloshin S. Overdiagnosed: making people sick in pursuit of health. Boston: Beacon Press, 2011.

3. Brodersen J, Schwartz LM, Heneghan C, et al. Overdiagnosis: what it is and what it isn't. BMJ Evid Based Med 2018;23:1 LP-3.

4. Cordoba G, Arnold S, Hansen MP. 6 Overdiagnosis and overtreatment of infectious diseases in general practice. How and where to break the endless loop? BMJ Evidence-Based Med 2018;23:A2-3.

5. Carter SM, Rogers W, Heath I, et al. The challenge of overdiagnosis begins with its definition. BMJ 2015;350:h869.

6. Spinks A, Glasziou PP DMCB. Antibiotics for sore throat (Review). Cochrane database Syst Rev 2013;11:CD000023.

7. Venekamp RP, Sanders SL, Glasziou PP, et al. Antibiotics for acute otitis media in children. Cochrane database Syst Rev 2015;6:CD000219.

8. Hulscher ME, van der Meer JW, Grol RP. Antibiotic use: how to improve it? Int J Med Microbiol 2010;300:351-6.
9. Urbiztondo I, de Oliveira S, Hernández-Flores N, et al. General practitioners' views on the acceptability and applicability of using quality indicators as an intervention to reduce unnecessary prescription of antibiotics in four South American Countries. Antibiotics 2018;7:57.

10. Tonkin-Crine S, Yardley L, Little P. Antibiotic prescribing for acute respiratory tract infections in primary care: a systematic review and metaethnography. J Antimicrob Chemother 2011;66:2215-23.

11. Fletcher-Lartey S, Yee M, Gaarslev C, et al. Why do general practitioners prescribe antibiotics for upper respiratory tract infections to meet patient expectations: a mixed methods study. BMJ Open 2016;6:e012244.

12. Gaarslev C, Yee M, Chan G, et al. A mixed methods study to understand patient expectations for antibiotics for an upper respiratory tract infection. Antimicrob Resist Infect Control 2016;5:39.

13. Cabral C, Lucas PJ, Ingram J, et al. "It's safer to ..." parent consulting and clinician antibiotic prescribing decisions for children with respiratory tract infections: An analysis across four qualitative studies. Soc Sci Med 2015;136-137:156-64.

14. Hutchinson JM, Jelinski S, Hefferton D, et al. Role of diagnostic labeling in antibiotic prescription. Can Fam Physician 2001;47:1217-24.

15. Llor C, Cots JM, Bjerrum L, et al. [Antibiotic prescribing in respiratory tract infections and predictive factors for their use]. Aten Primaria 2010;42:28-35.

16. Schmiemann G, Kniehl E, Gebhardt K, et al. The diagnosis of urinary tract infection: a systematic review. Dtsch Arztebl Int 2010;107:361-7.

17. Schot MJC, Dekker ARJ, Giorgi WG, et al. Diagnostic value of signs, symptoms and diagnostic tests for diagnosing pneumonia in ambulant children in developed countries: a systematic review. NPJ Prim Care Respir Med 2018;28:40.

18. Aalbers J, O'Brien KK, Chan WS, et al. Predicting streptococcal pharyngitis in adults in primary care: a systematic review of the diagnostic accuracy of symptoms and signs and validation of the centor score. BMC Med 2011;9:67.

19. Yang JH, Huang PY, Shie SS, et al. Predictive symptoms and signs of laboratory-confirmed influenza: a prospective surveillance study of two metropolitan areas in Taiwan. Medicine 2015;94:e1952.

20. Leeflang MM, Rutjes AW, Reitsma JB, et al. Variation of a test's sensitivity and specificity with disease prevalence. CMAJ 2013;185:E537-E544.

21. Caliendo AM, Gilbert DN, Ginocchio CC, et al. Better tests, better care: improved diagnostics for infectious diseases. Clin Infect Dis 2013;57(suppl 3):S139-70.

22. Aabenhus R, Jensen JUS, Jørgensen K, et al. Biomarkers as point-of-care tests to guide prescription of antibiotics in patients with acute respiratory infections in primary care. Cochrane Database Syst Rev 2014;11:CD010130.

23. Little P, Moore M, Hobbs FD, et al. PRImary care Streptococcal Management (PRISM) study: identifying clinical variables associated with Lancefield group A $\beta$-haemolytic streptococci and Lancefield non-Group A streptococcal throat infections from two cohorts of patients presenting with an acute sore throat. BMJ Open 2013;3:e003943.

24. Thompson M, Weigl B, Fitzpatrick A, et al. More than just accuracy: a novel method to incorporate multiple test attributes in evaluating diagnostic tests including point of care tests. IEEE J Transl Eng Health Med 2016;4:1-8.

25. Córdoba G, Holm A, Sørensen TM, et al. Use of diagnostic tests and the appropriateness of the treatment decision in patients with suspected urinary tract infection in primary care in Denmark - observational study. BMC Fam Pract 2018;19:65. 